\title{
Development and Validation of Retinal Vasculature Nomogram in Suspected Angina Due to Coronary Artery Disease
}

\author{
Pingting Zhong ${ }^{1,2}$, Jie Qin ${ }^{3}$, Zhixi Li ${ }^{4}$, Lei Jiang ${ }^{5}$, Qingsheng Peng ${ }^{1,2}$, Manqing Huang ${ }^{1}$, Yingwen Lin ${ }^{2,6}$, \\ Baoyi Liu ${ }^{1,7}$, Cong Li ${ }^{1,8}$, Qiaowei Wu ${ }^{1,7}$, Yu Kuang ${ }^{1}$, Shirong Cui ${ }^{9}$, Honghua Yu ${ }^{1}$, Zaiyi Liu ${ }^{10}$ and Xiaohong Yang ${ }^{1}$
}

Pingting Zhong, Jie Qin, and Zhixi Li contributed equally to this work.

Xiaohong Yang, Zaiyi Liu and Honghua Yu are joint senior authors.

${ }^{1}$ Guangdong Eye Institute, Department of Ophthalmology, Guangdong Provincial People’s Hospital, Guangdong Academy of Medical Sciences, Guangzhou, China.

${ }^{2}$ Shantou University Medical College, Shantou, China.

${ }^{3}$ School of Automation Science and Engineering, South China University of Technology, Guangzhou, China.

${ }^{4}$ State Key Laboratory of Ophthalmology, Zhongshan Ophthalmic Center, Sun Yat-sen University, Guangzhou, China.

${ }^{5}$ Guangdong Geriatrics Institute, Guangdong Provincial People’s Hospital, Guangdong Academy of Medical Sciences, Guangzhou, China.

${ }^{6}$ Department of Cardiology, Guangdong Cardiovascular Institute, Guangdong Provincial Key Laboratory of Coronary Heart Disease Prevention, Guangdong Provincial People’s Hospital, Guangdong Academy of Medical Sciences, Guangzhou, China.

${ }^{7}$ Southern Medical University, Guangzhou, China.

${ }^{8}$ School of Medicine, South China University of Technology, Guangzhou, China.

${ }^{9}$ Department of Statistics, University of California, Davis, CA, USA.

${ }^{10}$ Department of Radiology, Guangdong Provincial People’s Hospital, Guangdong Academy of Medical Sciences, Guangzhou, China.

Aims: To develop and validate a nomogram using retinal vasculature features and clinical variables to predict coronary artery disease (CAD) in patients with suspected angina.

Methods: The prediction model consisting of 795 participants was developed in a training set of 508 participants with suspected angina due to CAD, and data were collected from January 2018 to June 2019. The held-out validation was conducted with 287 consecutive patients from July 2019 to November 2019. All patients with suspected CAD received optical coherence tomography angiography (OCTA) examination before undergoing coronary CT angiography. LASSO regression model was used for data reduction and feature selection. Multivariable logistic regression analysis was used to develop the retinal vasculature model for predicting the probability of the presence of CAD.

Results: Three potential OCTA parameters including vessel density of the nasal and temporal perifovea in the superficial capillary plexus and vessel density of the inferior parafovea in the deep capillary plexus were further selected as independent retinal vasculature predictors. Model clinical electrocardiogram (ECG) OCTA (clinical variables + ECG + OCTA) was presented as the individual prediction nomogram, with good discrimination (AUC of 0.942 [95\% CI, 0.923-0.961] and 0.897 [95\% CI, 0.861-0.933] in the training and held-out validation sets, respectively) and good calibration. Decision curve analysis indicated the clinical applicability of this retinal vasculature nomogram.

Conclusions: The presented retinal vasculature nomogram based on individual probability can accurately identify the presence of $\mathrm{CAD}$, which could improve patient selection and diagnostic yield of aggressive testing before determining a diagnosis.

Key words: Coronary artery disease, Optical coherence tomography angiography, Retinal vasculature, Nomogram, Diagnosis, Prediction 


\section{Introduction}

Angina is the most common initial manifestation of coronary artery disease (CAD), and patients with new-onset angina might experience cardiac events in 1-2 years ${ }^{1)}$. Therefore, it is critical to early detect and timely manage the suspected angina due to $C A D^{2)}$. Great improvements had been made in clinical rapid assessments of suspected $\mathrm{CAD}^{3)}$, but many patients remained misdiagnosed. In the current context, more and more clinical trials have sought to streamline patient selection in identifying CAD. Several population-derived risk scores focused on the accuracy and comparability of coronary computed tomography angiography (CCTA) examination have thus been developed ${ }^{4-6)}$. Simple and accurate examinations continue to be needed in patient populations with suspected CAD before settling on a diagnosis.

Retinal vasculature holds sufficient information regarding vascular health ${ }^{7-10)}$, heart failure ${ }^{11)}$, hypertension ${ }^{12)}$, and subtypes of stroke ${ }^{13)}$. With the assistance of deep learning, recent studies have explored the value of retinal imaging in the prediction of cardiovascular risk factors ${ }^{14)}$, as well as the changes of retinal microvasculature affected by hypertension ${ }^{15)}$ and other chronic diseases ${ }^{16)}$. Besides, retinal vasculature was found to be associated with CAD, which could provide additional implications on cardiovascular risks coexisting with microvascular dysfunction in patients ${ }^{17,18)}$. However, these studies mostly focused on fundus photographs, which contained limited measurable information on retinal vasculature. Optical coherence tomography angiography (OCTA) is an automatic ophthalmic technique used to noninvasively visualize and quantitatively measure the ocular structures. An optimal approach with the implementation of retinal vasculature features, measured by OCTA examination, to explore the availability and clinical usefulness in predicting CAD has yet to be developed.

\section{Aim}

Therefore, in this cross-sectional study, we aimed to develop and validate a nomogram on the basis of quantified retinal vasculature features among patients with suspected angina due to CAD. If this model could be clinically used to predict the presence of $\mathrm{CAD}$, it might benefit the medical community in terms of optimizing patient selection. In the meantime, it might also improve the diagnostic yield and cost-effectiveness of CCTA or coronary angiography (CAG) examinations.

\section{Materials and Methods}

\section{Study Population}

This was a cross-sectional study mainly conducted in the Department of Cardiology, Guangdong Provincial People's Hospital-a tertiary hospital and key cardiovascular institute in South China. The study was approved by the Research Ethics Committee of the hospital (No. GDREC2019554H(R1)) and was performed according to the Declaration of Helsinki. Patient consent was obtained prior to the study.

The study was designed to collect demographic, clinical, and imaging data for patients with suspected chest pain due to $\mathrm{CAD}$ referred by primary-care cardiologists. All the participants underwent CCTA examinations. The confirmation of CAD on CCTA examination referred to the presence of lumen diameter reduction $\geq 50 \%$ in at least one major coronary artery. Overall, from January 2018 to June 2019, our training set consecutively included 508 participants: 281 patients with CAD and 227 participants without CAD. Of the 508 participants, 346 were males and 162 females, with a mean age of 59 years. From July 2019 to November 2019, a heldout validation set study was performed using the same inclusion criteria as the training set, resulting in 287 participants: 140 patients with CAD and 147 participants without CAD. Of the 287 participants, 176 were males and 111 females, with a mean age of 59 years. All the included participants received an OCTA examination before the CCTA procedure.

Exclusion criteria included patients with 1) preexisting hypertensive or diabetic retinopathy (HR or DR, respectively) based on the medical history provided and the clinical examination results of slitlamp biomicroscopy, indirect ophthalmoscopy, two 45-degree mydriatic colour fundus photographs, and OCTA. 2) uncooperating with ophthalmic examinations; or 3) moderate or high refractive error ( $\geq \pm 3$ dioptres). Patients with prior CAD and revascularisation were also excluded.

\section{Clinical Data}

A structural interview before OCTA and CCTA

Address for correspondence: Xiaohong Yang, Guangdong Provincial People's Hospital, Guangdong Academy of Medical Sciences, Guangzhou 510080, China E-mail: syyangxh@scut.edu.cn

Received: November 17, 2020 Accepted for publication: February 2, 2021

Copyright@2021 Japan Atherosclerosis Society

This article is distributed under the terms of the latest version of CC BY-NC-SA defined by the Creative Commons Attribution License. 
examinations was conducted to collect baseline data on the presence of cardiovascular risk factors. The participants' age, sex, body mass index, and history of hypertension and diabetes mellitus - as well as the baseline cholesterol values-were documented. A resting electrocardiogram (ECG) was performed for all patients on admission, which was recorded as normal or abnormal depending on the manifestation of regional change in ST segment or $\mathrm{T}$ wave. The thresholds for laboratory risk factors used in stratified analyses were defined according to the guidelines on the management of dyslipidemia ${ }^{19)}$.

\section{Retinal Image Acquisition and Assessment}

OCTA (RTVue-XR Avanti; Optovue, Fremont, CA, USA) is a fast ( $<1$ minute per eye) ophthalmic examination with a $840-\mathrm{nm}$-wavelength spectraldomain OCT that generates $304 \times 304$ scans in 2.9 seconds at a speed of $70 \mathrm{kHz}^{20,21)}$. The device uses a split-spectrum amplitude-decorrelation angiography algorithm $^{22)}$

Specifically, in this study, OCTA data consisted of the vasculature features of the macular and disc area. The vessel density (VD) of the superficial capillary plexus (SCP), deep capillary plexus (DCP), foveal density (FD-300), and radial peripapillary capillary (RPC) was measured. Further, the retinal nerve fiber layer (RNFL) thickness was quantified. Descriptions of the OCTA examination and retinal vasculature features are shown in the Supplemental Material and Supplemental Fig. 1.

\section{CCTA Procedure and Assessment}

CCTA image acquisition and processing were performed according to the local protocol. All the participants were scheduled to undergo a $\geq$ 64-detector row CCTA evaluation. All coronary scans were analyzed by two experienced cardiologists blinded to the adjunctive investigations and retinal assessment results.

\section{Machine Learning}

The patients included in our analyses were characterized by ECG assessment and a total of nine readily available demographic clinical variables. A machine learning (ML) algorithm was constructed to differentiate patients with CAD from those without $\mathrm{CAD}$. The ML technique was implemented using the R software (version 3.6.3; http://www.Rproject.org).

In the training data set, the least absolute shrinkage and selection operator (LASSO) method (glmnet) was employed to select the most predictive clinical variables and OCTA parameters according to the strength of the univariable association with outcomes ${ }^{23)}$. The "rms" package was used to create models in order to classify patients with suspected CAD. The training set was created using 10-fold cross-validation. Briefly, the data set was randomly and equally divided into 10 folds, followed by the performance of 10 validation experiments. Each fold took turns to be the testing set, and the remaining nine folds were regarded as the training sets. In the end, the overall performance was provided on the basis of the combination of 10 experimental models. The ultimate point of this cross-validation was to obtain a more accurate estimate of model prediction.

\section{Statistical Analysis}

The mean \pm standard deviation was used to present quantitative variables with normal distributions, median (interquartile ranges) to present quantitative variables with abnormal distributions, and percentages to describe categorical variables.

OCTA data and clinical variables were selected by LASSO. The performance of Model OCTA was compared with the performance of Model ECG - the primary screening method for suspected CAD. Besides, to create a better model in predicting CAD, clinical variables selected by LASSO and ECG assessment were added to Model OCTA. This study aimed to provide a clinical quantitative tool for doctors to predict the individual probability of the presence of CAD. A nomogram was, therefore, built on the basis of the results of multivariable logistic regression analysis in the training set. Calibration analyses by the rms package were applied to evaluate the calibration of the nomogram. The nomogram was then applied to all the participants in the held-out validation set, with the total points for each participant calculated.

Decision curve analysis (DCA) by the "rmda" package was conducted in the held-out validation data set to assess the clinical application of the nomogram, where the individual net benefits at different threshold probabilities were calculated.

The classification performances of the models were reported using the area under the curve (AUC) and the associated 95\% confidence interval (CI). All statistical tests with $p<0.05$ were considered significant.

\section{Results}

\section{Clinical Characteristics}

The flowchart of participant selection is shown in Supplemental Fig.2, and the characteristics of the two data sets are presented in Table 1 . The presence of CAD was $281(55.3 \%)$ and $140(48.8 \%)$ in the 
Table 1. Baseline characteristics of the participants in training and held-out validation sets $(n=795)$

\begin{tabular}{|c|c|c|c|c|c|c|}
\hline & \multicolumn{2}{|c|}{ Training set $(n=508)$} & \multirow[b]{2}{*}{$p$-value } & \multicolumn{2}{|c|}{ Held-out validation set $(n=287)$} & \multirow[b]{2}{*}{$p$-value } \\
\hline & $\begin{array}{c}\text { Non-CAD } \\
\text { cohort }(n=227)\end{array}$ & $\begin{array}{l}\text { CAD cohort } \\
(n=281)\end{array}$ & & $\begin{array}{c}\text { Non-CAD } \\
\text { cohort }(n=147)\end{array}$ & $\begin{array}{l}\text { CAD cohort } \\
(n=140)\end{array}$ & \\
\hline \multicolumn{7}{|l|}{ Cardiovascular characteristics } \\
\hline Age (years), mean $\pm S D$ & $57.3 \pm 10.4$ & $59.8 \pm 9.4$ & $0.005^{*}$ & $59.2 \pm 11.3$ & $60.1 \pm 9.7$ & 0.576 \\
\hline Sex, $n(\%)$ & & & $<0.001^{*}$ & & & $<0.001^{*}$ \\
\hline Male & $121(53.3)$ & $225(80.1)$ & & $65(44.2)$ & $111(79.3)$ & \\
\hline Female & $106(46.7)$ & $56(19.9)$ & & $82(55.8)$ & $29(20.7)$ & \\
\hline BMI, $n(\%)$ & & & $0.046^{*}$ & & & $0.008^{*}$ \\
\hline$<25 \mathrm{~kg} / \mathrm{m}^{2}$ & $159(70.0)$ & $173(61.6)$ & & $118(80.3)$ & $93(66.4)$ & \\
\hline$\geq 25 \mathrm{~kg} / \mathrm{m}^{2}$ & $68(30.0)$ & $108(38.4)$ & & $29(19.7)$ & $47(33.6)$ & \\
\hline Hypertension, $n(\%)$ & & & $0.008^{*}$ & & & 0.759 \\
\hline Without & $62(27.3)$ & $108(38.4)$ & & $51(34.7)$ & $51(36.4)$ & \\
\hline With & $165(72.7)$ & $173(61.6)$ & & $96(65.3)$ & $89(63.6)$ & \\
\hline Diabetic mellitus, $n(\%)$ & & & $0.032^{*}$ & & & 0.067 \\
\hline Without & $166(73.1)$ & $228(81.1)$ & & $124(84.4)$ & $106(75.7)$ & \\
\hline With & $61(26.9)$ & $53(18.9)$ & & $23(15.6)$ & $34(24.3)$ & \\
\hline \multicolumn{7}{|l|}{ Cholesterol levels } \\
\hline Triglycerides, $n(\%)$ & & & $0.001^{*}$ & & & $0.001^{*}$ \\
\hline$\leq 1.7 \mathrm{mmol} / \mathrm{L}$ & $176(77.5)$ & $181(64.4)$ & & $116(78.9)$ & $86(61.4)$ & \\
\hline$>1.7 \mathrm{mmol} / \mathrm{L}$ & $51(22.5)$ & $100(35.6)$ & & $31(21.1)$ & $54(38.6)$ & \\
\hline Total cholesterol, $n(\%)$ & & & $<0.001^{*}$ & & & 0.102 \\
\hline$<4.1 \mathrm{mmol} / \mathrm{L}$ & $144(63.4)$ & $122(43.4)$ & & $88(59.9)$ & $82(58.6)$ & \\
\hline$\geq 4.1$ and $\leq 5.2 \mathrm{mmol} / \mathrm{L}$ & $31(13.7)$ & $90(32.0)$ & & $23(15.6)$ & $34(24.3)$ & \\
\hline$>5.2 \mathrm{mmol} / \mathrm{L}$ & $52(22.9)$ & $69(24.6)$ & & $36(24.5)$ & $24(17.1)$ & \\
\hline HDL-C, $n(\%)$ & & & $<0.001^{*}$ & & & $<0.001^{*}$ \\
\hline$\leq 1.0 \mathrm{mmol} / \mathrm{L}$ & $4(1.8)$ & $151(53.7)$ & & $13(8.8)$ & $75(53.6)$ & \\
\hline$>1.0 \mathrm{mmol} / \mathrm{L}$ & $223(98.2)$ & $130(46.3)$ & & $134(91.2)$ & $65(46.4)$ & \\
\hline LDL-C, $n(\%)$ & & & $<0.001^{*}$ & & & $<0.001^{*}$ \\
\hline$<1.8 \mathrm{mmol} / \mathrm{L}$ & $5(2.2)$ & $26(9.3)$ & & $3(2.0)$ & $17(12.1)$ & \\
\hline$\geq 1.8$ and $<2.6 \mathrm{mmol} / \mathrm{L}$ & $24(10.6)$ & $84(29.8)$ & & $16(10.9)$ & $45(32.2)$ & \\
\hline$\geq 2.6$ and $<3.0 \mathrm{mmol} / \mathrm{L}$ & $4(1.7)$ & $44(15.7)$ & & $10(6.8)$ & $20(14.3)$ & \\
\hline$\geq 3.0 \mathrm{mmol} / \mathrm{L}$ & $194(85.5)$ & $127(45.2)$ & & $118(80.3)$ & $58(41.4)$ & \\
\hline ECG, $n(\%)$ & & & $<0.001^{*}$ & & & $<0.001^{*}$ \\
\hline Normal & $222(97.8)$ & $168(59.8)$ & & $143(97.3)$ & $87(62.1)$ & \\
\hline Abnormal & $5(2.2)$ & $113(40.2)$ & & $4(2.7)$ & $53(37.9)$ & \\
\hline \multicolumn{7}{|l|}{ VD in the SCP } \\
\hline Perifovea-tempo & $46.38 \pm 4.44$ & $45.73 \pm 3.95$ & 0.082 & $46.69 \pm 4.68$ & $44.20 \pm 4.33$ & $<0.001^{*}$ \\
\hline Perifovea-nasal & $53.86 \pm 4.17$ & $52.83 \pm 3.52$ & $0.003^{*}$ & $53.66 \pm 4.23$ & $51.95 \pm 4.44$ & $0.001^{*}$ \\
\hline \multicolumn{7}{|l|}{ VD in the DCP } \\
\hline Parafovea-inferior & $55.07 \pm 5.23$ & $50.94 \pm 5.36$ & $<0.001^{*}$ & $54.98 \pm 4.87$ & $49.39 \pm 5.87$ & $<0.001^{*}$ \\
\hline Perifovea-superior & $52.93 \pm 7.39$ & $48.75 \pm 6.92$ & $<0.001^{*}$ & $52.68 \pm 7.17$ & $47.50 \pm 7.17$ & $<0.001^{*}$ \\
\hline Superior-hemi & $52.12 \pm 6.22$ & $48.35 \pm 5.72$ & $<0.001^{*}$ & $51.94 \pm 6.19$ & $47.19 \pm 5.56$ & $<0.001^{*}$ \\
\hline
\end{tabular}

Abbreviations: CAD, coronary artery disease; BMI, body mass index; HDL-C, high-density lipoprotein cholesterol; LDL-C, low-density lipoprotein cholesterol; ECG, electrocardiogram; SCP, superficial capillary plexus; DCP, deep capillary plexus.

Continuous variables are displayed as mean \pm standard deviation; categorical data are displayed as numbers (percentages).

The thresholds for total cholesterol were 4.1 and $5.2 \mathrm{mmol} / \mathrm{L}$; the triglyceride value that defined hypertriglyceridemia was $>1.7 \mathrm{mmol} / \mathrm{L}$, and the threshold for HDL-C was $1.0 \mathrm{mmol} / \mathrm{L}$. LDL-C was the primary target for cardiovascular disease prevention in different risk stratifications, with the goals of $<1.8 \mathrm{mmol} / \mathrm{L}$ for the very high-risk group, $<2.6 \mathrm{mmol} / \mathrm{L}$ for the high-risk group, and $<3.0 \mathrm{mmol} / \mathrm{L}$ for the low- to moderate-risk group. ${ }^{*}<0.05$ is considered statistically significant. 


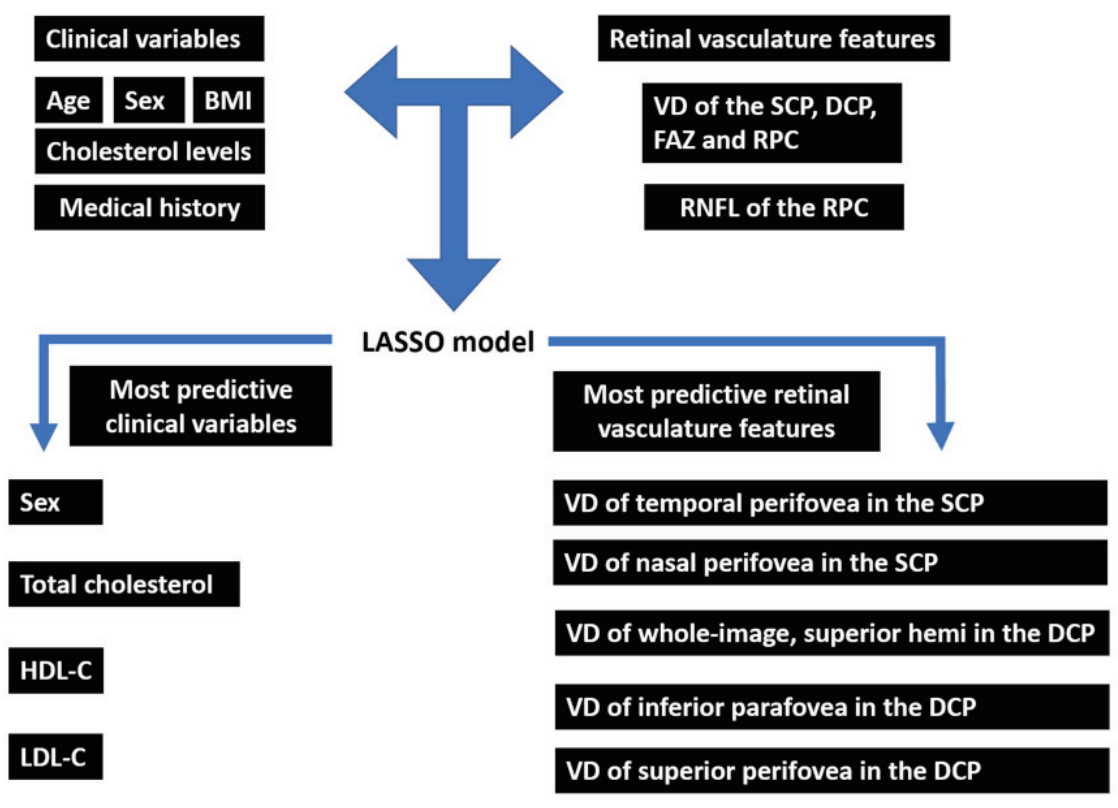

Fig. 1. Flow chart of feature selection

Abbreviation: BMI, body mass index; SCP, superficial capillary plexus; DCP, deep capillary plexus; RPC, radial peripapillary capillary; RNFL, retinal never fiber layer; VD, vessel density; HDL-C, high-density lipoprotein cholesterol; LDL-C, low-density lipoprotein cholesterol.

training and held-out validation sets, respectively. The presence of an abnormal resting ECG was 118 $(23.2 \%)$ and $57(19.9 \%)$ in the training and held-out validation sets, respectively. Although there was a temporal disconnect, most of the clinical variables did not show significant differences between the training and held-out validation sets or within the non-CAD and CAD groups (see Supplemental Table 1). The analyses of retinal vasculature features among the study groups are provided in Supplemental Table 2.

\section{Feature Selection}

A total of 71 retinal vasculature features were reduced to five potential predictors (VD of the nasal and temporal perifovea in the SCP and VD of the inferior parafovea, superior perifovea, and wholeimage, superior hemi in the DCP); and a total of nine clinical variables were reduced to four potential predictors (sex, total cholesterol, LDL-C and HDL-C) on the basis of 508 participants in the training set (see Fig. 1 and Supplemental Fig. 3).

\section{Development, Apparent Performances, and Clinical Application of An Individual Prediction Model \\ Multivariable logistic regression analysis began with above four clinical variables, ECG assessment and five OCTA parameters, and then identified four clinical variables (sex, total cholesterol, LDL-C and}

HDL-C), ECG assessment, and three potential OCTA parameters (VD of the nasal and temporal perifovea in the SCP and VD of the inferior parafovea in the DCP) as independent predictors (Table 2). The Model clinical ECG OCTA that incorporated the selected independent predictors was presented as the retinal vasculature nomogram (Fig. 2).

The calibration curves for the probability of $\mathrm{CAD}$ using this nomogram indicated good agreement between the prediction and observation in both the training and held-out validation sets (Supplemental Fig.4).

Model ECG exhibited good performance in terms of predicting individuals with $\mathrm{CAD}$, with an AUC of 0.690 (95\% CI, 0.660-0.720) and 0.677 (95\% CI, 0.634-0.720) in the training and held-out validation sets, respectively. Model OCTA exhibited the better performance than Model ECG in terms of predicting individuals with CAD, with an AUC of 0.758 (95\% CI, 0.716-0.799) and 0.730 (95\% CI, $0.673-0.788)$ in the training and held-out validation sets, respectively. With the addition of common clinical variables assessment into Model ECG and Model OCTA, the performance of Model clinical ECG (ECG + clinical variables) to predict CAD was significantly improved, with an AUC of $0.914(95 \%$ CI, 0.891-0.937) and 0.850 (95\% CI, 0.805-0.895) in the training and held-out validation sets, 
Table 2. Risk factors for predicting coronary artery disease in the nomogram

\begin{tabular}{lccc}
\hline Intercept and variables & $\beta$ & OR $(95 \%$ CI $)$ & $p$-value \\
\hline Intercept & 16.816 & & $<0.001^{*}$ \\
Sex & -0.890 & $0.41(0.30-0.57)$ & $<0.001^{*}$ \\
ECG & 1.898 & $6.67(4.53-9.97)$ & $<0.001^{*}$ \\
Total cholesterol & 0.953 & $2.59(2.12-3.20)$ & $<0.001^{*}$ \\
HDL-C & -4.009 & $0.02(0.01-0.04)$ & $<0.001^{*}$ \\
LDL-C & -0.806 & $0.45(0.37-0.54)$ & $<0.001^{*}$ \\
Temporal perifovea in the SCP & 0.154 & $1.17(1.09-1.25)$ & $<0.001^{*}$ \\
Nasal perifovea in the SCP & -0.124 & $0.88(0.82-0.95)$ & $<0.001^{*}$ \\
Inferior parafovea in the DCP & -0.165 & $0.85(0.82-0.88)$ & $<0.001^{*}$ \\
C index & & & \\
Training set & & $0.942(0.923-0.961)$ & \\
Held-out validation set & & $0.897(0.861-0.933)$ & \\
\hline
\end{tabular}

Abbreviations: OR, odds ratio; CI, confidence interval; HDL-C, high-density lipoprotein cholesterol; LDL-C, low-density lipoprotein cholesterol; SCP, superficial capillary plexus; DCP, deep capillary plexus.

$\beta$ is the regression coefficient. Cholesterol values are presented as categorical variables, and the retinal vasculature features are presented as continuous variables.

${ }^{*} p<0.05$ is considered statistically significant.

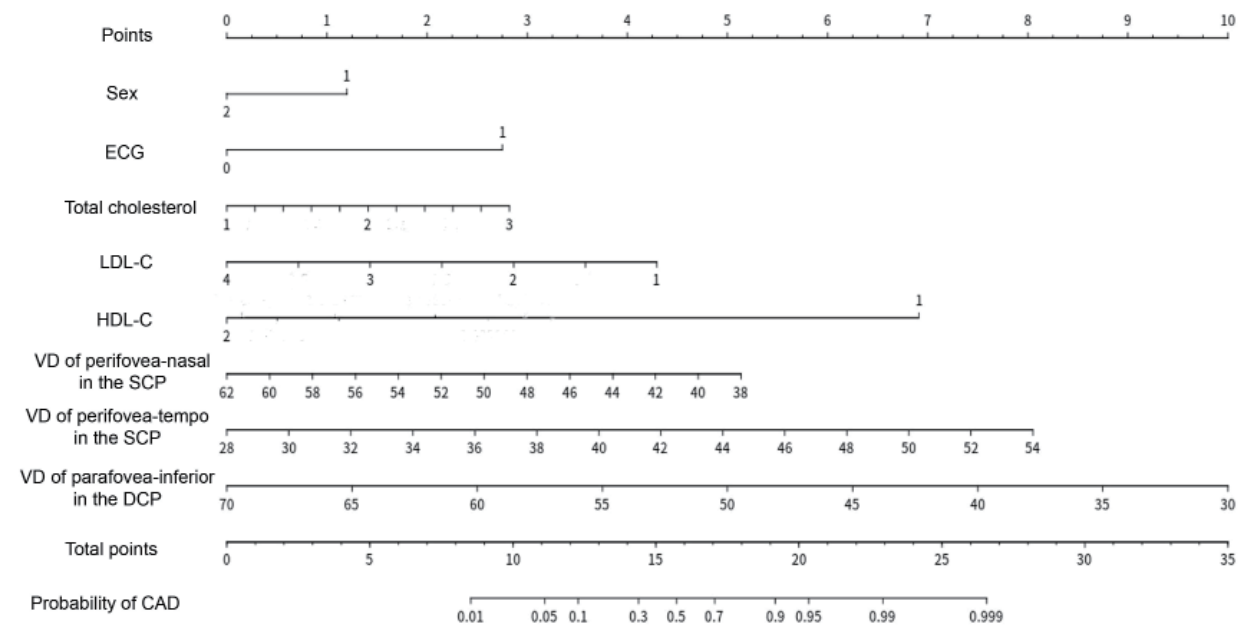

Fig.2. Developed retinal vasculature nomogram

The clinical variables were presented as categorical variables. Number signature definition: Sex: $1=$ male and $2=$ female; Total cholesterol: $1=>5.2 \mathrm{mmol} / \mathrm{L}, 2=\geq 4.1$ and $\leq 5.2 \mathrm{mmol} / \mathrm{L}$, and $3=<4.1 \mathrm{mmol} / \mathrm{L}$; LDL$\mathrm{C}: 1=<1.8 \mathrm{mmol} / \mathrm{L}, 2=\geq 1.8$ and $<2.6 \mathrm{mmol} / \mathrm{L}, 3=\geq 2.6$ and $<3.0 \mathrm{mmol} / \mathrm{L}$, and $4=\geq 3.0 \mathrm{mmol} / \mathrm{L}$; HDL-C: $1=\leq 1.0 \mathrm{mmol} / \mathrm{L}$ and $2=>1.0 \mathrm{mmol} / \mathrm{L}$.

The results of ECG assessment were presented as categorical variables: $0=$ normal and $1=$ abnormal.

The retinal vasculature features were presented as continuous variables.

Abbreviations: ECG, electrocardiogram; LDL-C, low-density lipoprotein cholesterol; HDL-C, high-density lipoprotein cholesterol; VD, vessel density; SCP, superficial capillary plexus; DCP, deep capillary plexus.

respectively; the performance of Model clinical OCTA (OCTA + clinical variables) to predict CAD was significantly improved, with an AUC of 0.910 (95\% CI, $0.887-0.934)$ and 0.862 (95\% CI, 0.819-0.905) in the training and held-out validation sets, respectively. The Model clinical ECG OCTA (nomogram) performed best in discriminating CAD from non-CAD participants, with an AUC of 0.942
(95\% CI, 0.923-0.961) and 0.897 (95\% CI, 0.8610.933 ) in the training and held-out validation sets, respectively. The sensitivity and specificity in predicting CAD of these models are shown in Fig. 3.

The DCA for this retinal vasculature nomogram is presented in Fig.4. A model is considered clinically useful when it has the highest net benefit across the threshold range, for which an individual is designated

\section{Advance Publication Journal of Atherosclerosis and Thrombosis}




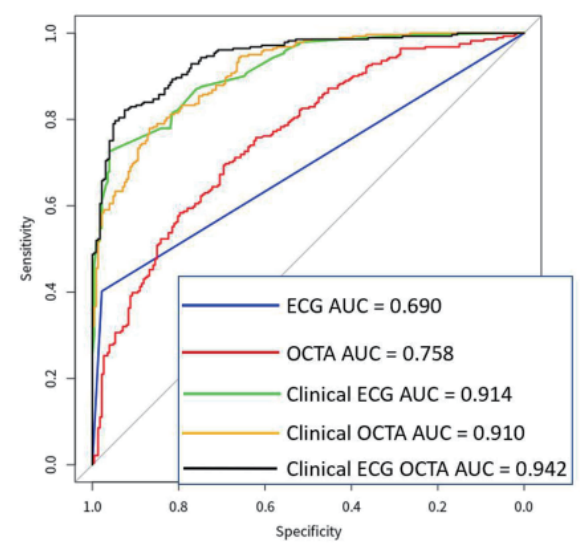

(A) Training set

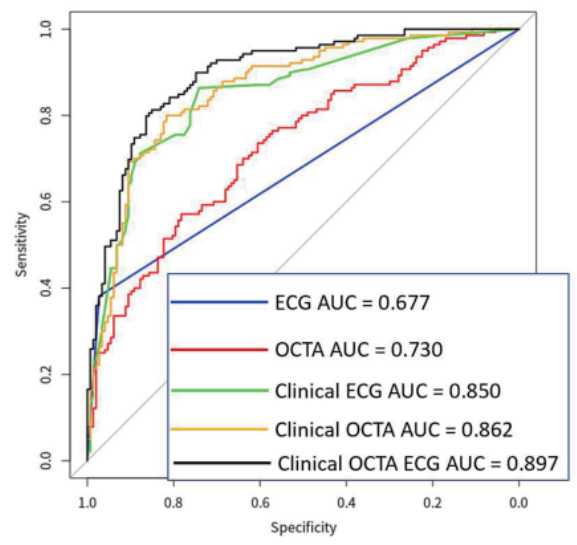

(B) Held-out validation set

\begin{tabular}{|l|c|c|c|c|}
\hline & \multicolumn{2}{|c|}{ Training set } & \multicolumn{2}{c|}{ Held-out validation set } \\
\hline & Sensitivity & Specificity & Sensitivity & Specificity \\
\hline Model ECG & 0.402 & 0.978 & 0.381 & 0.973 \\
\hline Model OCTA & 0.694 & 0.696 & 0.571 & 0.782 \\
\hline Model clinical ECG & 0.726 & 0.960 & 0.800 & 0.816 \\
\hline Model clinical OCTA & 0.779 & 0.868 & 0.800 & 0.816 \\
\hline Model clinical ECG OCTA & 0.822 & 0.925 & 0.813 & 0.850 \\
\hline
\end{tabular}

Fig. 3. Discrimination curves of the prediction models in two data sets

ROC curves for Model ECG, Model OCTA, Model clinical OCTA, Model clinical ECG, and Model clinical ECG OCTA (nomogram) in differentiating patients with $\mathrm{CAD}$ from those without $\mathrm{CAD}$ in the $(\mathrm{A})$ training and $(\mathrm{B})$ held-out validation sets.

Model clinical OCTA includes four clinical variables (sex, total cholesterol, HDL-C and LDL-C) and five OCTA parameters (VD of the nasal and temporal perifovea in the SCP and VD of the inferior parafovea, superior perifovea, and whole-image, superior hemi in the DCP).

Model clinical ECG includes four clinical variables (sex, total cholesterol, HDL-C and LDL-C) and ECG assessments.

Model clinical ECG OCTA (nomogram) includes four clinical variables (sex, total cholesterol, HDL-C and LDL-C), ECG assessments, and three OCTA parameters (VD of the nasal and temporal perifovea in the SCP and VD of the inferior parafovea in the DCP).

Abbreviations: ROC, receiver operator characteristic curve; OCTA, optical coherence tomography angiography; ECG, electrocardiogram; $\mathrm{CAD}$, coronary artery disease; VD, vessel density; SCP, superficial capillary plexus; DCP, deep capillary plexus; HDL-C, high-density lipoprotein cholesterol; LDL-C, low-density lipoprotein cholesterol.

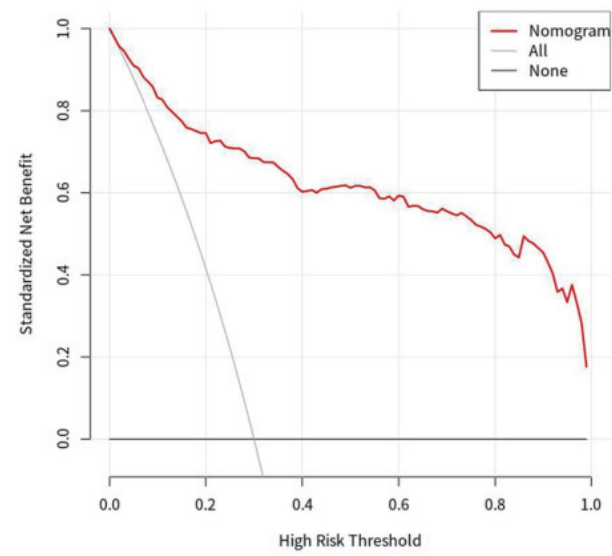

Fig. 4. Decision curve analysis for the nomogram

The Y-axis measures the net benefit. The red line represents the retinal vasculature nomogram. The gray line represents the assumption that all patients have CAD. The black line represents the assumption that no patient presents with CAD. The decision curve indicated that using the retinal vasculature nomogram in the current study to predict CAD could add more benefit than the receipt of cardiac examinations (such as CCTA or CAG) or no intervention.

Abbreviations: CAD, coronary artery disease; CCTA, coronary computed tomography angiography; CAG, coronary angiography.

\section{Advance Publication Journal of Atherosclerosis and Thrombosis}


at high risk. Our decision curve showed that the threshold probability of a patient or doctor was $0 \%$, which indicated that using this nomogram to predict CAD could add more benefits compared to either receiving or not receiving cardiac examination.

\section{Discussion}

In this study, a diagnostic nomogram was developed and validated to identify CAD in patients with suspected angina. This nomogram incorporated retinal vasculature features, ECG assessment, and clinical variables showed the best performance in predicting CAD. A total of 71 candidate OCTA features were decreased to five potential independent predictors by the LASSO method. The implementation of the ML technique in the clinical setting could optimize the process of selecting appropriate candidates and improve the diagnostic yield of aggressive testing before determining a diagnosis. This developed nomogram also provided the individual probability of the presence of CAD in suspected patients. Such an easy-to-use nomogram might contribute to the prediction of CAD.

Overusing imaging modalities to diagnose CAD is a frequent phenomenon because of the fear of undertreatment. There has been an ongoing need to find a validated diagnostic model with optimal performance to direct downstream testing for CAD. In recent years, $M L$ techniques have brought about many breakthroughs in classification tasks and emerged as highly effective methods for making predictions and performing decision-making in a number of disciplines ${ }^{24)}$. Typically, ML allows computer algorithms to build a model from test in order to predict future outcomes on the basis of new input data ${ }^{25)}$. It has gradually become a popular option and powerful tool across the cardiovascular domain ${ }^{26,27)}$. With the implementation of ML, this study developed a nomogram using OCTA parameters and clinical risk factors to predict the individual probability of the presence of CAD.

The retinal vasculature features used in the prediction models showed considerable discrimination. With the assistance of deep learning, retinal images have recently been assessed for corresponding changes under the condition of hypertension ${ }^{15)}$, hyperglycemia, and dyslipidemia ${ }^{16)}$. Retinal imaging was also considered a potential biomarker in the assessment of cardiovascular diseases. A study has used fundus photographs via deep learning to predict cardiovascular risk factors, such as age, sex, systolic blood pressure, and major adverse cardiac events ${ }^{14)}$. Another study has also explored the value of retinal images in predicting atherosclerosis ${ }^{28)}$. Specifically, retinal vasculature features hold valuable and sufficient information to detect the development and progression of $\mathrm{CAD}^{8)}$, which began with prior discoveries where patients with CAD were found to present with narrowing retinal arterioles ${ }^{29}, 30$ ) and decreased retinal $\mathrm{VD}^{31,32)}$. As previously documented, the narrowing retinal arterioles were associated with a greater likelihood of obstructive stenosis $(\geq 50 \%)$ in at least one major coronary artery ${ }^{33)}$. A hypothesis has been put forth that an independent association between retinal arteriolar narrowing and incident CAD was the microvascular process ${ }^{34)}$. The decreased retinal VD could be associated with the narrowing retinal arterioles, which might result from microvascular damage - such as the process of aging and hypertension-and from intimal thickening, medial hyperplasia, hyalinization, and sclerosis ${ }^{34)}$. Besides, the decreased retinal VD could also result from endothelial dysfunction coexisted in CAD patients. Endothelial dysfunction is a systemic condition and has been detected in the coronary epicardial as well as in peripheral arteries ${ }^{35)}$, in which peripheral endothelial function was found to be associated with coronary artery endothelial function ${ }^{36)}$. The utility of retinal vasculature could significantly improve the estimate of the probability of CAD. OCTA is a noninvasive and measurable technique with multiple pieces of information on quantified retinal vasculature, which might provide more detailed messages in the assessment and prediction of cardiovascular diseases.

The discrimination performance of Model OCTA was better than Model ECG-a common and primary screening method in identifying patients with suspected angina due to CAD. The resting ECG was characterized by low sensitivity in detecting CAD before undergoing subsequent aggressive cardiac examinations and so was exercise $\mathrm{ECG}^{37)}$. A recent study has reported the limited incremental value of ECG in assessing CAD, and therefore more effective methods of risk stratification were requested ${ }^{38)}$. Our presented retinal vasculature nomogram shows the potential to reduce the need for further stress testing, improve the diagnostic yield, and increase feasibility for the diagnosis of CAD.

The presented nomogram was characterized by several strengths: First, the nomogram revealed a considerable ability to reach comparable discrimination performances, which indicated the potential of retinal vasculature in the assessment for suspected CAD patient. Second, ML algorithms are more adaptive than traditional statistical in managing complex medical data ${ }^{39)}$. ML algorithms split input 
space into different cells based on every feature, which allows the algorithms to learn non-linear relation of data ${ }^{40)}$. On the contrary, traditional statistical analysis assume the linear separability of the input space of data and thus attempts to find a hyperplane to cut the input space into two half-spaces, which makes it unable to deliver such approximation for complex data analysis ${ }^{40)}$. In our study, both clinical data and ophthalmic data are easily obtained. Besides, LASSO method is commonly used and good at selecting the most predictive variables according to the strength of the univariable association with outcomes ${ }^{23)}$. Third, it could reduce radiation exposure and associated cardiac examination costs. Both CCTA and CAG are regarded as primce methods for accurately diagnosing CAD. However, most patients found with minimal or without CAD were generally not suitable for such aggressive procedures. The SCOT-HEART trial revealed that only $25 \%$ of suspected patients undergoing CCTA examination were diagnosed with obstructive CAD ${ }^{41)}$. Data from the National Cardiovascular Data Registry also indicated a low diagnostic yield of invasive CAG with $37.6 \%$ patients having obstructive CAD, regardless of the precatheterization rate of $83.9 \%$ in noninvasive testing ${ }^{42)}$. Other concerns about CCTA or CAG examinations have also been raised in terms of their radiation exposure and potential poor image quality in patients with obesity, arrhythmia, or coronary calcification ${ }^{43}{ }^{44)}$. Hence, there is a need for clinically based models that can predict CAD and direct downstream aggressive testing. In recent years, emphasis has been placed on risk stratification and pretest probability methods in diagnosing CAD. For example, a study has compared the pretest probability of CAD between the Diamond and Forrester (DF) score and two CAD consortium scores recommended by the European Society of Cardiology. Their results showed the AUC of 0.713 versus 0.752 and 0.791 for the DF versus the other two CAD consortium models, respectively ${ }^{6}$. Another comparable study that applied coronary artery calcium scoring has showed great improvements in discrimination using the ML (with AUC increasing from 0.773 to 0.881 ) and CAD consortium models (with AUC increasing from 0.734 to 0.866$)^{45)}$. Alternatively, a nomogram consisting of easily measured retinal vasculature features, clinical variables and ECG assessment was developed and validated in the current study.

The clinical application of this nomogram is the most important argument based on the interpretation of an individual benefit with additional examination or care. In the current study, the DCA was plotted, which could offer novel insights into clinical outcomes on the basis of threshold probabilities. The results of the DCA revealed significant benefits of using the presented nomogram to predict the presence of CAD. The study suggests that this retinal vasculature nomogram could be a follow-up tool and gatekeeper to identify low-risk individuals before moving to further diagnostic testing. However, it is yet to determine whether a simple model that applied retinal vasculature features, ECG assessment, and clinical variables is preferable for use in the domain of assessment for CAD. Support from prospective randomized trials is needed to evaluate the effectiveness of such an approach.

We acknowledge several limitations in the current study: First, because of technique limitations, not all retinal vascular parameters - such as choroidal VD and thickness - could be collected in the study. Second, only a small sample of participants were included; and selection bias might exist in the participants recruitment. Further, data analysis from multicenter research would be better for this study; however, being a large cardiac center in South China, most patients with CAD are usually transferred here. Further extensive and prospective studies are needed to enhance and validate the clinical utility of this model.

\section{Conclusions}

A nomogram combining retinal vasculature features, ECG assessment, and clinical variables was developed and validated to predict CAD. OCTA is a noninvasive and novel ophthalmic examination, which could be suggested as a screening and follow-up tool for patients with suspected angina due to CAD prior to aggressive diagnostic testing. This nomogram suits the need for preventive therapy and improves patient selection and diagnostic yield of aggressive testing before determining the diagnosis.

\section{Acknowledgements}

The authors have no acknowledgements.

\section{Disclosures}

The authors declare that there is no conflict of interest.

\section{Funding Sources}

This work was supported by Science and Technology Program of Guangzhou, China (202002020049) (Xiaohong Yang); Project of 
Investigation on Health Status of Employees in Financial Industry in Guangzhou (Z012014075) (Xiaohong Yang); The National Natural Science Foundation of China (81870663) (Honghua Yu); The Talent Introduction Fund of Guangdong Provincial People's Hospital (KJ012019087, Y012018145) (Honghua $\mathrm{Yu}$ ); Medical Scientific Research Foundation of Guangdong Province of China (C2019044) (Manqing Huang).

\section{Authors' Contributions}

Z.PT, Q. J, and L. ZX contributed equally to this work. Y. XH had full access to all the data in the study and take responsibility for the integrity of the data and the accuracy of the data analysis. Conception and design: Z. PT, Q. J, L. ZX, Y. HH, L. ZY, and Y. $\mathrm{XH}$. Acquisition, analysis, or interpretation of data: All authors. Drafting of the manuscript: Z. PT, Q. J, and L. ZX. Critical revision of manuscript for important intellectual analysis: All authors. Statistical analysis: Z. PT, C. SR, and Y. XH. Final approval of the version to be published: Z. PT, Q. J, L. ZX, Y. $\mathrm{HH}, \mathrm{L} . \mathrm{ZY}$, and Y. XH. All authors revised the article critically for important intellectual content. All authors have read and approved the final manuscript.

\section{References}

1) Gandhi MM, Lampe FC, Wood DA. Incidence, clinical characteristics, and short-term prognosis of angina pectoris. Br Heart J, 1995; 73: 193-198

2) Kravitz RL, Laouri M, Kahan JP, Guzy P, Sherman T, Hilborne L, Brook RH. Validity of criteria used for detecting underuse of coronary revascularization. JAMA, 1995; 274: 632-638

3) Sekhri N, Feder GS, Junghans C, Hemingway H, Timmis AD. How effective are rapid access chest pain clinics? Prognosis of incident angina and non-cardiac chest pain in 8762 consecutive patients. Heart, 2007; 93: 458-463

4) Douglas PS, Hoffmann U, Patel MR, Mark DB, Al-Khalidi HR, Cavanaugh B, Cole J, Dolor RJ, Fordyce CB, Huang M, Khan MA, Kosinski AS, Krucoff MW, Malhotra V, Picard MH, Udelson JE, Velazquez EJ, Yow E, Cooper LS, Lee KL. Outcomes of anatomical versus functional testing for coronary artery disease. N. Engl. J. Med., 2015; 372: 1291-1300

5) Genders TS, Steyerberg EW, Alkadhi H, Leschka S, Desbiolles L, Nieman K, Galema TW, Meijboom WB, Mollet NR, de Feyter PJ, Cademartiri F, Maffei E, Dewey M, Zimmermann E, Laule M, Pugliese F, Barbagallo R, Sinitsyn V, Bogaert J, Goetschalckx K, Schoepf UJ, Rowe GW, Schuijf JD, Bax JJ, de Graaf FR, Knuuti J, Kajander $S$, van Mieghem CA, Meijs MF, Cramer MJ, Gopalan D, Feuchtner G, Friedrich G, Krestin GP, Hunink MG. A clinical prediction rule for the diagnosis of coronary artery disease: validation, updating, and extension. Eur. Heart J.,
2011; 32: 1316-1330

6) Bittencourt MS, Hulten E, Polonsky TS, Hoffman U, Nasir K, Abbara S, Di Carli M, Blankstein R. European Society of Cardiology-Recommended Coronary Artery Disease Consortium Pretest Probability Scores More Accurately Predict Obstructive Coronary Disease and Cardiovascular Events Than the Diamond and Forrester Score: The Partners Registry. Circulation, 2016; 134: 201-211

7) Bhargava M, Ikram MK, Wong TY. How does hypertension affect your eyes? J Hum Hypertens, 2012; 26: $71-83$

8) Flammer J, Konieczka K, Bruno RM, Virdis A, Flammer AJ, Taddei S. The eye and the heart. Eur. Heart J., 2013; 34: 1270-1278

9) Grassi G, Buzzi S, Dell'Oro R, Mineo C, Dimitriadis K, Seravalle G, Lonati L, Cuspidi C. Structural alterations of the retinal microcirculation in the "prehypertensive" highnormal blood pressure state. Curr. Pharm. Des., 2013; 19: 2375-2381

10) Wong TY. Is retinal photography useful in the measurement of stroke risk? Lancet Neurol, 2004; 3: 179183

11) Nägele MP, Barthelmes J, Ludovici V, Cantatore S, von Eckardstein A, Enseleit F, Lüscher TF, Ruschitzka F, Sudano I, Flammer AJ. Retinal microvascular dysfunction in heart failure. Eur. Heart J., 2018; 39: 47-56

12) Cheung CY, Ikram MK, Sabanayagam C, Wong TY. Retinal microvasculature as a model to study the manifestations of hypertension. Hypertension, 2012; 60: 1094-1103

13) Doubal FN, MacGillivray TJ, Hokke PE, Dhillon B, Dennis MS, Wardlaw JM. Differences in retinal vessels support a distinct vasculopathy causing lacunar stroke. Neurology, 2009; 72: 1773-1778

14) Poplin R, Varadarajan AV, Blumer K, Liu Y, McConnell MV, Corrado GS, Peng L, Webster DR. Prediction of cardiovascular risk factors from retinal fundus photographs via deep learning. Nat Biomed Eng, 2018; 2: 158-164

15) Dai G, He W, Xu L, Pazo EE, Lin T, Liu S, Zhang C. Exploring the effect of hypertension on retinal microvasculature using deep learning on East Asian population. PLoS ONE, 2020; 15: e0230111

16) Zhang L, Yuan M, An Z, Zhao X, Wu H, Li H, Wang Y, Sun B, Li H, Ding S, Zeng X, Chao L, Li P, Wu W. Prediction of hypertension, hyperglycemia and dyslipidemia from retinal fundus photographs via deep learning: A cross-sectional study of chronic diseases in central China. PLoS ONE, 2020; 15: e0233166

17) Taqueti VR, Di Carli MF. Coronary Microvascular Disease Pathogenic Mechanisms and Therapeutic Options: JACC State-of-the-Art Review. J. Am. Coll. Cardiol., 2018; 72: 2625-2641

18) Gupta A, Taqueti VR, van de Hoef TP, Bajaj NS, Bravo PE, Murthy VL, Osborne MT, Seidelmann SB, Vita T, Bibbo CF, Harrington M, Hainer J, Rimoldi O, Dorbala S, Bhatt DL, Blankstein R, Camici PG, Di Carli MF. Integrated Noninvasive Physiological Assessment of Coronary Circulatory Function and Impact on Cardiovascular Mortality in Patients With Stable 
Coronary Artery Disease. Circulation, 2017; 136: 23252336

19) Catapano AL, Graham I, De Backer G, Wiklund O, Chapman MJ, Drexel H, Hoes AW, Jennings CS, Landmesser U, Pedersen TR, Reiner Ž, Riccardi G, Taskinen MR, Tokgozoglu L, Verschuren WMM, Vlachopoulos C, Wood DA, Zamorano JL, Cooney MT. 2016 ESC/EAS Guidelines for the Management of Dyslipidaemias. Eur. Heart J., 2016; 37: 2999-3058

20) Spaide RF, Klancnik JM, Cooney MJ. Retinal vascular layers imaged by fluorescein angiography and optical coherence tomography angiography. JAMA Ophthalmol, 2015; 133: 45-50

21) Agemy SA, Scripsema NK, Shah CM, Chui T, Garcia PM, Lee JG, Gentile RC, Hsiao YS, Zhou Q, Ko T, Rosen RB. Retinal Vascular Perfusion Density Mapping Using Optical Coherence Tomography Angiography in Normals and Diabetic Retinopathy Patients. Retina, 2015; 35: 2353-2363

22) Motaghiannezam R, Fraser S. Logarithmic intensity and speckle-based motion contrast methods for human retinal vasculature visualization using swept source optical coherence tomography. Biomed Opt Express, 2012; 3: 503-521

23) Ambler G, Seaman S, Omar RZ. An evaluation of penalised survival methods for developing prognostic models with rare events. Stat Med, 2012; 31: 1150-1161

24) Deo RC. Machine Learning in Medicine. Circulation, 2015; 132: 1920-1930

25) Waljee AK, Higgins PD. Machine learning in medicine: a primer for physicians. Am. J. Gastroenterol., 2010; 105: 1224-1226

26) Motwani M, Dey D, Berman DS, Germano G, Achenbach S, Al-Mallah MH, Andreini D, Budoff MJ, Cademartiri F, Callister TQ, Chang HJ, Chinnaiyan K, Chow BJ, Cury RC, Delago A, Gomez M, Gransar H, Hadamitzky M, Hausleiter J, Hindoyan N, Feuchtner G, Kaufmann PA, Kim YJ, Leipsic J, Lin FY, Maffei E, Marques H, Pontone G, Raff G, Rubinshtein R, Shaw LJ, Stehli J, Villines TC, Dunning A, Min JK, Slomka PJ. Machine learning for prediction of all-cause mortality in patients with suspected coronary artery disease: a 5-year multicentre prospective registry analysis. Eur. Heart J., 2017; 38: 500-507

27) van Rosendael AR, Maliakal G, Kolli KK, Beecy A, Al'Aref SJ, Dwivedi A, Singh G, Panday M, Kumar A, Ma X, Achenbach S, Al-Mallah MH, Andreini D, Bax JJ, Berman DS, Budoff MJ, Cademartiri F, Callister TQ, Chang HJ, Chinnaiyan K, Chow BJW, Cury RC, DeLago A, Feuchtner G, Hadamitzky M, Hausleiter J, Kaufmann PA, Kim YJ, Leipsic JA, Maffei E, Marques H, Pontone G, Raff GL, Rubinshtein R, Shaw LJ, Villines TC, Gransar H, Lu Y, Jones EC, Peña JM, Lin FY, Min JK. Maximization of the usage of coronary CTA derived plaque information using a machine learning based algorithm to improve risk stratification; insights from the CONFIRM registry. J Cardiovasc Comput Tomogr, 2018; 12: 204-209

28) Chang J, Ko A, Park SM, Choi S, Kim K, Kim SM, Yun JM, Kang U, Shin IH, Shin JY, Ko T, Lee J, Oh BL, Park KH. Association of Cardiovascular Mortality and Deep
Learning-Funduscopic Atherosclerosis Score derived from Retinal Fundus Images. Am. J. Ophthalmol., 2020

29) Seidelmann SB, Claggett B, Bravo PE, Gupta A, Farhad H, Klein BE, Klein R, Di Carli M, Solomon SD. Retinal Vessel Calibers in Predicting Long-Term Cardiovascular Outcomes: The Atherosclerosis Risk in Communities Study. Circulation., 2016; 134: 1328-1338

30) Gopinath B, Chiha J, Plant AJ, Thiagalingam A, Burlutsky G, Kovoor P, Liew G, Mitchell P. Associations between retinal microvascular structure and the severity and extent of coronary artery disease. Atherosclerosis., 2014; 236(1): 25-30

31) Arnould L, Guenancia C, Azemar A, Alan G, Pitois S, Bichat F, Zeller M, Gabrielle PH, Bron AM, CreuzotGarcher C, Cottin Y. The EYE-MI Pilot Study: A Prospective Acute Coronary Syndrome Cohort Evaluated With Retinal Optical Coherence Tomography Angiography. Invest Ophthalmol Vis Sci., 2018; 59(10): 4299-4306

32) Wang J, Jiang J, Zhang Y, Qian YW, Zhang JF, Wang ZL. Retinal and choroidal vascular changes in coronary heart disease: an optical coherence tomography angiography study. Biomed Opt Express., 2019; 10(4): 1532-1544

33) Tabatabaee A, Asharin MR, Dehghan MH, Pourbehi MR, Nasiri-Ahmadabadi M, Assadi M. Retinal vessel abnormalities predict coronary artery diseases. Perfusion, 2013; 28: 232-237

34) Wong TY, Klein R, Sharrett AR, Duncan BB, Couper DJ, Tielsch JM, Klein BE, Hubbard LD. Retinal arteriolar narrowing and risk of coronary heart disease in men and women. The Atherosclerosis Risk in Communities Study. JAMA, 2002; 287: 1153-1159

35) Anderson TJ, Gerhard MD, Meredith IT, Charbonneau F, Delagrange D, Creager MA, Selwyn AP, Ganz P. Systemic nature of endothelial dysfunction in atherosclerosis. Am. J. Cardiol., 1995; 75: 71B-74B

36) Anderson TJ, Uehata A, Gerhard MD, Meredith IT, Knab S, Delagrange D, Lieberman EH, Ganz P, Creager $\mathrm{MA}$, Yeung AC. Close relation of endothelial function in the human coronary and peripheral circulations. J. Am. Coll. Cardiol., 1995; 26: 1235-1241

37) Singh T, Bing R, Dweck MR, van Beek EJR, Mills NL, Williams MC, Villines TC, Newby DE, Adamson PD. Exercise Electrocardiography and Computed Tomography Coronary Angiography for Patients With Suspected Stable Angina Pectoris: A Post Hoc Analysis of the Randomized SCOT-HEART Trial. JAMA Cardiol, 2020

38) Sekhri N, Feder GS, Junghans C, Eldridge S, Umaipalan A, Madhu R, Hemingway $\mathrm{H}$, Timmis AD. Incremental prognostic value of the exercise electrocardiogram in the initial assessment of patients with suspected angina: cohort study. BMJ, 2008; 337: a2240

39) Lapuschkin S, Wäldchen S, Binder A, Montavon G, Samek W, Müller KR. Unmasking Clever Hans predictors and assessing what machines really learn. Nat Commun, 2019; 10: 1096

40) LeCun Y, Bengio Y, Hinton G. Deep learning. Nature, 2015; 521: 436-444

41) SCOT-HEART investigators. CT coronary angiography in patients with suspected angina due to coronary heart disease (SCOT-HEART): an open-label, parallel-group, 
multicentre trial. Lancet, 2015; 385: 2383-2391

42) Patel MR, Peterson ED, Dai D, Brennan JM, Redberg RF, Anderson HV, Brindis RG, Douglas PS. Low diagnostic yield of elective coronary angiography. $\mathrm{N}$. Engl. J. Med., 2010; 362: 886-895

43) Miller JM, Rochitte CE, Dewey M, Arbab-Zadeh A, Niinuma H, Gottlieb I, Paul N, Clouse ME, Shapiro EP, Hoe J, Lardo AC, Bush DE, de Roos A, Cox C, Brinker J, Lima JA. Diagnostic performance of coronary angiography by 64-row CT. N. Engl. J. Med., 2008; 359: 2324-2336

44) Mowatt G, Cummins E, Waugh N, Walker S, Cook J, Jia $\mathrm{X}$, Hillis GS, Fraser C. Systematic review of the clinical effectiveness and cost-effectiveness of 64-slice or higher computed tomography angiography as an alternative to invasive coronary angiography in the investigation of coronary artery disease. Health Technol Assess, 2008; 12: iii-iv, ix-143

45) Al'Aref SJ, Maliakal G, Singh G, van Rosendael AR, Ma $\mathrm{X}, \mathrm{Xu} \mathrm{Z}$, Alawamlh OAH, Lee B, Pandey M, Achenbach S, Al-Mallah MH, Andreini D, Bax JJ, Berman DS, Budoff MJ, Cademartiri F, Callister TQ, Chang HJ, Chinnaiyan K, Chow BJW, Cury RC, DeLago A, Feuchtner G, Hadamitzky M, Hausleiter J, Kaufmann PA, Kim YJ, Leipsic JA, Maffei E, Marques H, Gonçalves PA, Pontone G, Raff GL, Rubinshtein R, Villines TC, Gransar H, Lu Y, Jones EC, Peña JM, Lin FY, Min JK, Shaw LJ. Machine learning of clinical variables and coronary artery calcium scoring for the prediction of obstructive coronary artery disease on coronary computed tomography angiography: analysis from the CONFIRM registry. Eur. Heart J., 2020; 41: 359-367 


\section{Supplemental Methods}

Description of OCTA Examination and Images Assessment

Participants had their pupil dilated and received an optical coherence tomography angiography (OCTA) examination performed by trained ophthalmologists. A $6 \times 6 \mathrm{~mm}^{2}$ macular highdefinition (HD) scan mode and a $4.5 \times 4.5 \mathrm{~mm}^{2}$ disc HD were collected within one minute for each participant in the study. Both eyes of the participants were examined by OCTA, but only the data from one eye were used for analyses-for participants born in even-numbered years, it was the right eye, and for those born in odd-numbered years, it was the left eye. Only images with a quality index $\geq 6 / 10$ were retained.

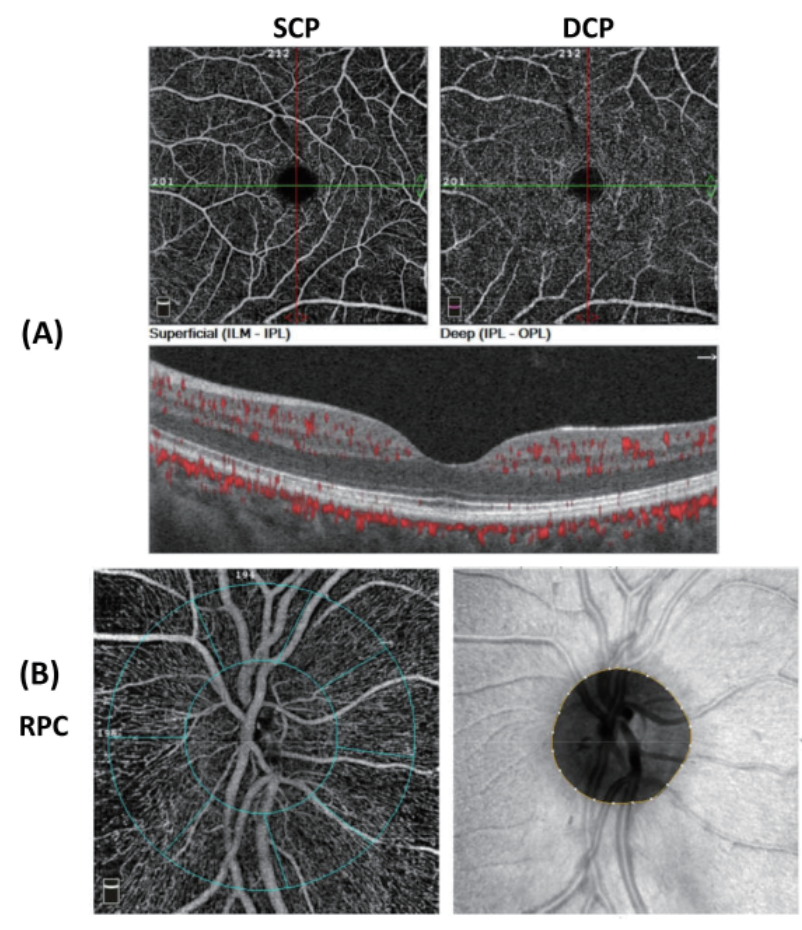

Non-CAD

\section{Description of Retinal Vasculature Features}

SCP refers to a slab extending from the internal limiting membrane (ILM) to $10 \mu \mathrm{m}$ above the inner plexiform layer (IPL), while the DCP slab extends from $10 \mu \mathrm{m}$ above the IPL to $10 \mu \mathrm{m}$ below the outer plexiform layer (OPL). FD-300 is a parameter demonstrating the capillary density from ILM to OPL in a 300- $\mu \mathrm{m}$-wide region around the foveal avascular zone (FAZ). Parafovea refers to the area inside the 1-3 $\mathrm{mm}$ concentric ring center of the fovea, while the perifovea refers to the actual $3-5 \mathrm{~mm}$ concentric ring center. RPC is a slab extending from the ILM to the RNFL that fits a circle $(2 \mathrm{~mm}$ in diameter) centered on the optic disc. The peripapillary region is defined as a $1-2 \mathrm{~mm}$ round annulus around the optic disc. The peripapillary VD and RNFL thickness were quantified in the RPC segment. All VD and thickness of RNFL were automatically measured by the OCTA software.

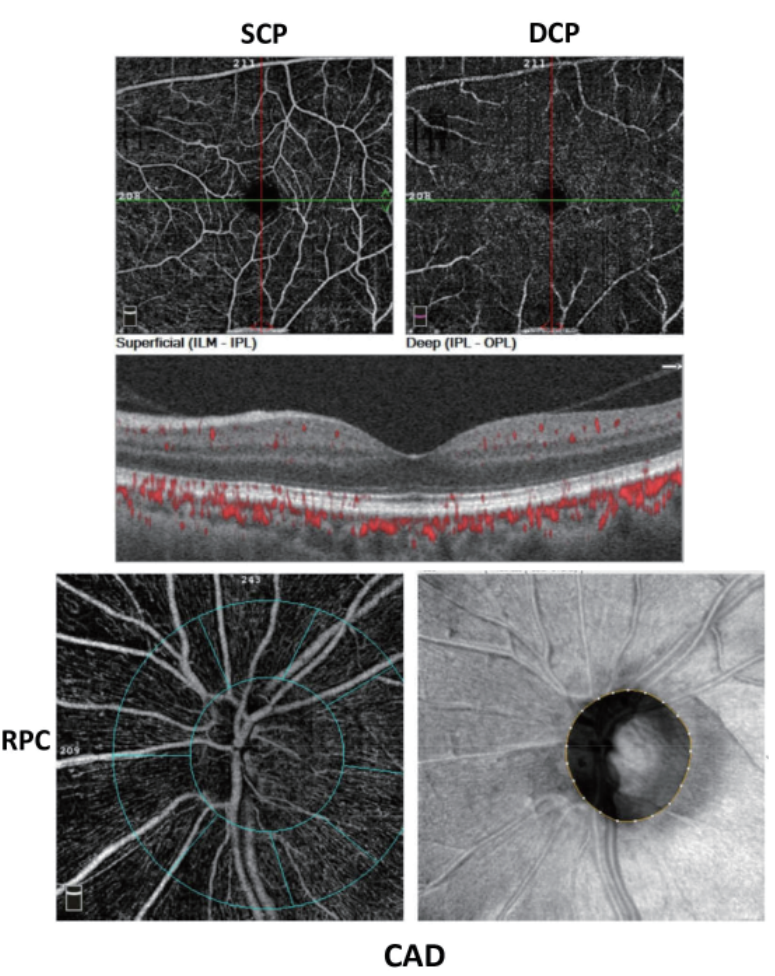

Supplemental Fig. 1. Example OCT angiograms of vessel density measurement in the non-CAD and CAD participants

(A) Different layers of the retina demonstrated by OCTA, including the superficial capillary plexus (SCP), deep capillary plexus (DCP) and avascular zones.

(B) Retinal thickness and vessel density in the radial peripapillary capillary (RPC). 

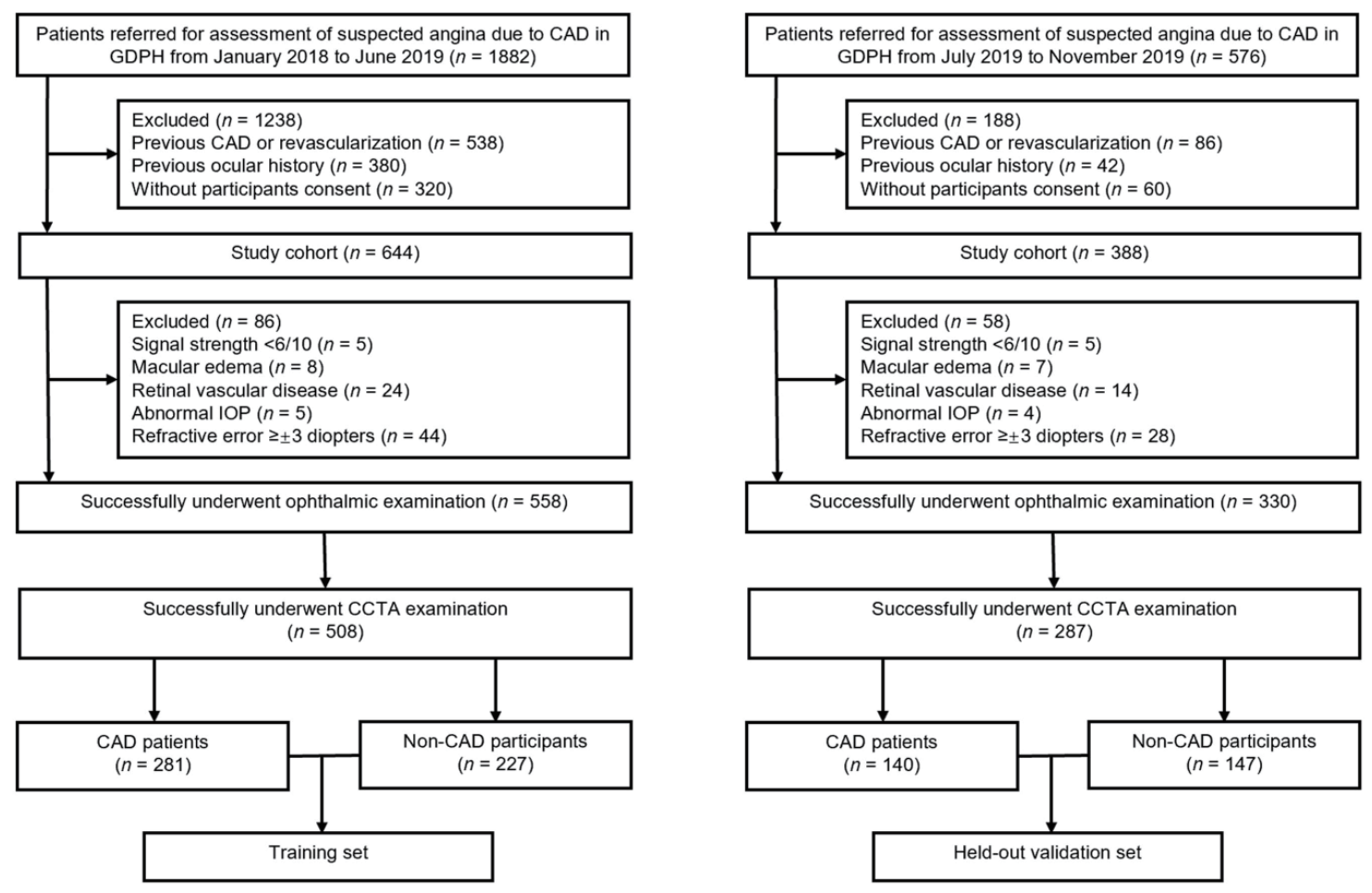

Supplemental Fig.2. Flow chart of participant selection

Abbreviation: CAD, coronary artery disease; CCTA, coronary CT angiography; GDPH, Guangdong Provincial People’s Hospital. 
Supplemental Table 1. Baseline characteristics of participants in the training and held-out validation sets $(n=795)$

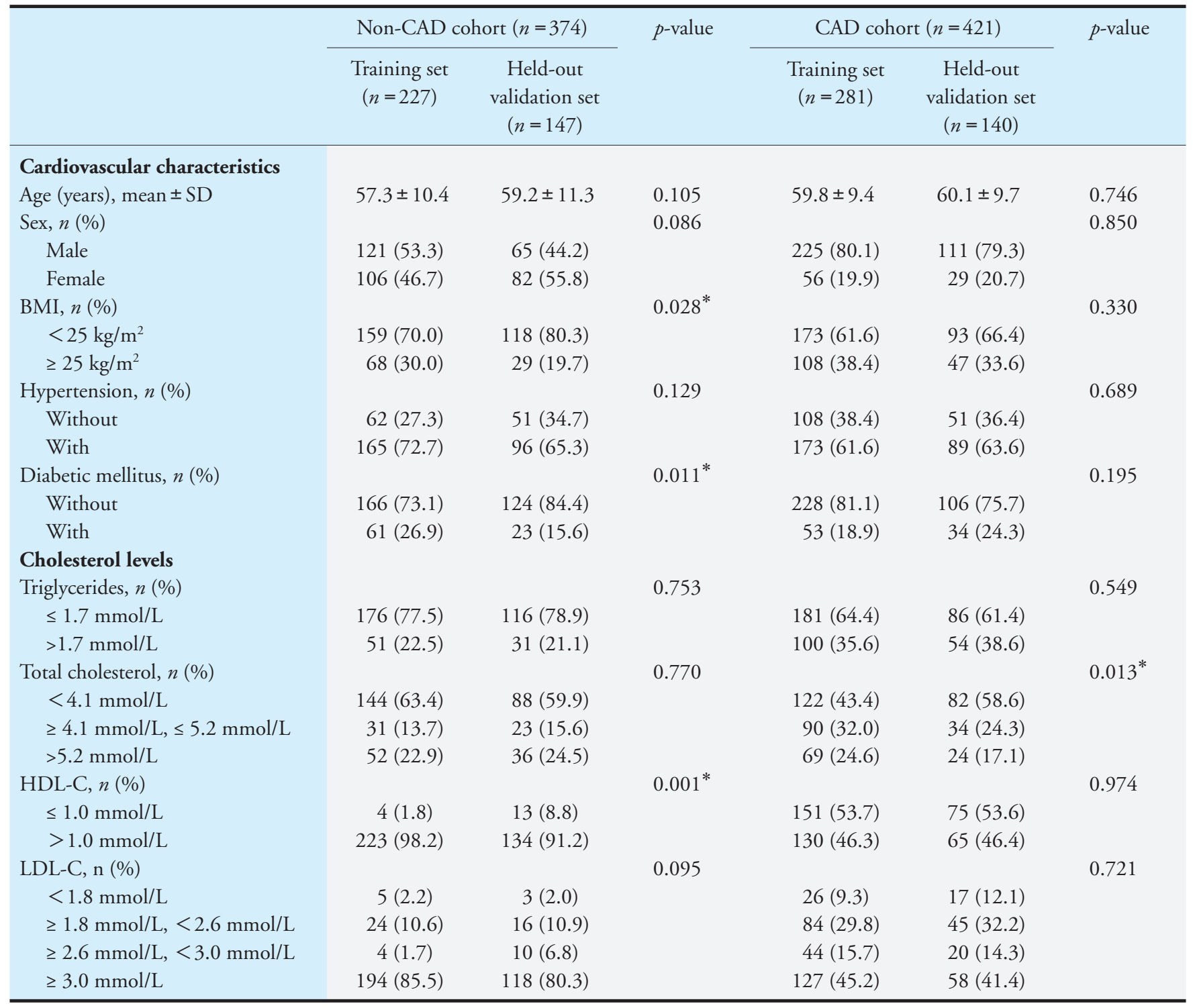

Abbreviations: CAD, coronary artery disease; BMI, body mass index; HDL-C, high-density lipoprotein cholesterol; LDL-C, low-density lipoprotein cholesterol.

Continuous variable displayed as mean $\pm \mathrm{SD}$; categorical data are displayed as number (percentage).

${ }_{p}^{*}<0.05$ is considered statistically significant. 
Supplemental Table 2. Retinal vasculature features in the participants $(n=795)$

\begin{tabular}{|c|c|c|c|c|c|c|}
\hline & \multicolumn{3}{|c|}{ Training set $(n=508)$} & \multicolumn{3}{|c|}{ Held-out validation set $(n=287)$} \\
\hline & $\begin{array}{c}\text { Non-CAD } \\
\text { cohort }(n=227)\end{array}$ & $\begin{array}{l}\text { CAD cohort } \\
(n=281)\end{array}$ & $p$-value & $\begin{array}{c}\text { Non-CAD } \\
\text { cohort }(n=147)\end{array}$ & $\begin{array}{l}\text { CAD cohort } \\
(n=140)\end{array}$ & $p$-value \\
\hline \multicolumn{7}{|c|}{ VD of SCP $(\%)$, mean \pm SD } \\
\hline Whole-image & $49.38 \pm 4.10$ & $48.71 \pm 3.39$ & $0.046^{*}$ & $49.61 \pm 4.18$ & $47.68 \pm 3.94$ & $<0.001^{*}$ \\
\hline Superior-hemi & $49.62 \pm 4.24$ & $48.94 \pm 3.36$ & $0.042 *$ & $49.89 \pm 4.17$ & $47.95 \pm 4.00$ & $<0.001^{*}$ \\
\hline Inferior-hemi & $49.12 \pm 4.12$ & $48.48 \pm 3.59$ & 0.064 & $49.30 \pm 4.32$ & $47.37 \pm 4.08$ & $<0.001^{*}$ \\
\hline Fovea & $17.91 \pm 7.37$ & $18.26 \pm 6.69$ & 0.571 & $18.48 \pm 7.29$ & $17.67 \pm 7.36$ & 0.350 \\
\hline Parafovea & $51.70 \pm 4.88$ & $50.97 \pm 3.86$ & 0.060 & $51.99 \pm 4.99$ & $49.74 \pm 4.94$ & $<0.001^{*}$ \\
\hline Parafovea-superior-hemi & $51.79 \pm 5.18$ & $51.23 \pm 3.88$ & 0.164 & $52.16 \pm 5.02$ & $50.05 \pm 5.06$ & $<0.001^{*}$ \\
\hline Parafovea-inferior-hemi & $51.61 \pm 5.00$ & $50.71 \pm 4.20$ & $0.027^{*}$ & $51.81 \pm 5.27$ & $49.43 \pm 5.11$ & $<0.001^{*}$ \\
\hline Parafovea-tempo & $51.72 \pm 4.84$ & $51.10 \pm 4.15$ & 0.122 & $51.70 \pm 5.01$ & $50.16 \pm 4.71$ & $0.008^{*}$ \\
\hline Parafovea-superior & $52.46 \pm 5.88$ & $51.91 \pm 4.15$ & 0.224 & $52.79 \pm 5.46$ & $50.46 \pm 5.77$ & $<0.001^{*}$ \\
\hline Parafovea-nasal & $50.58 \pm 5.00$ & $50.07 \pm 4.47$ & 0.218 & $51.21 \pm 5.34$ & $48.97 \pm 5.63$ & $0.001^{*}$ \\
\hline Parafovea-inferior & $52.04 \pm 5.89$ & $50.79 \pm 4.79$ & $0.009^{*}$ & $52.24 \pm 5.67$ & $49.39 \pm 5.54$ & $<0.001^{*}$ \\
\hline Perifovea & $50.15 \pm 4.28$ & $49.28 \pm 3.57$ & $0.012^{*}$ & $50.29 \pm 4.24$ & $48.12 \pm 4.15$ & $<0.001^{*}$ \\
\hline Perifovea-superior-hemi & $50.34 \pm 4.41$ & $49.48 \pm 3.60$ & $0.015^{*}$ & $50.59 \pm 4.24$ & $48.37 \pm 4.28$ & $<0.001^{*}$ \\
\hline Perifovea-inferior-hemi & $49.97 \pm 4.30$ & $49.08 \pm 3.75$ & $0.014^{*}$ & $49.98 \pm 4.44$ & $47.87 \pm 4.23$ & $<0.001^{*}$ \\
\hline Perifovea-tempo & $46.38 \pm 4.44$ & $45.73 \pm 3.95$ & 0.082 & $46.69 \pm 4.68$ & $44.20 \pm 4.33$ & $<0.001^{*}$ \\
\hline Perifovea-superior & $50.49 \pm 4.74$ & $49.44 \pm 3.84$ & $0.006^{*}$ & $50.72 \pm 4.46$ & $48.49 \pm 4.49$ & $<0.001^{*}$ \\
\hline Perifovea-nasal & $53.86 \pm 4.17$ & $52.83 \pm 3.52$ & $0.003^{*}$ & $53.66 \pm 4.23$ & $51.95 \pm 4.44$ & $0.001^{*}$ \\
\hline Perifovea-inferior & $49.95 \pm 4.72$ & $49.15 \pm 4.13$ & $0.044^{*}$ & $50.16 \pm 4.56$ & $47.93 \pm 4.37$ & $<0.001^{*}$ \\
\hline \multicolumn{7}{|c|}{ VD of DCP $(\%)$, mean \pm SD } \\
\hline Whole-image & $51.68 \pm 6.15$ & $47.89 \pm 5.57$ & $<0.001^{*}$ & $51.48 \pm 6.14$ & $46.62 \pm 5.33$ & $<0.001^{*}$ \\
\hline Superior-hemi & $52.12 \pm 6.22$ & $48.35 \pm 5.72$ & $<0.001^{*}$ & $51.94 \pm 6.19$ & $47.19 \pm 5.56$ & $<0.001^{*}$ \\
\hline Inferior-hemi & $51.22 \pm 6.27$ & $47.42 \pm 5.64$ & $<0.001^{*}$ & $51.00 \pm 6.32$ & $45.99 \pm 5.42$ & $<0.001^{*}$ \\
\hline Fovea & $32.80 \pm 8.62$ & $32.62 \pm 7.43$ & 0.799 & $33.62 \pm 8.19$ & $31.84 \pm 8.56$ & 0.074 \\
\hline Parafovea & $55.70 \pm 4.53$ & $52.70 \pm 4.32$ & $<0.001^{*}$ & $56.11 \pm 4.26$ & $51.68 \pm 4.37$ & $<0.001^{*}$ \\
\hline Parafovea-superior-hemi & $55.76 \pm 5.03$ & $53.27 \pm 4.35$ & $<0.001^{*}$ & $56.28 \pm 4.39$ & $52.42 \pm 4.17$ & $<0.001^{*}$ \\
\hline Parafovea-inferior-hemi & $55.64 \pm 4.56$ & $52.13 \pm 4.58$ & $<0.001^{*}$ & $55.93 \pm 4.32$ & $50.93 \pm 4.90$ & $<0.001^{*}$ \\
\hline Parafovea-tempo & $56.42 \pm 4.71$ & $53.64 \pm 4.37$ & $<0.001^{*}$ & $56.72 \pm 4.60$ & $52.81 \pm 4.09$ & $<0.001^{*}$ \\
\hline Parafovea-superior & $55.21 \pm 5.71$ & $52.62 \pm 4.77$ & $<0.001^{*}$ & $55.76 \pm 4.87$ & $51.46 \pm 4.93$ & $<0.001^{*}$ \\
\hline Parafovea-nasal & $56.10 \pm 4.48$ & $53.59 \pm 4.60$ & $<0.001^{*}$ & $56.96 \pm 4.10$ & $53.06 \pm 4.65$ & $<0.001^{*}$ \\
\hline Parafovea-inferior & $55.07 \pm 5.23$ & $50.94 \pm 5.36$ & $<0.001^{*}$ & $54.98 \pm 4.87$ & $49.39 \pm 5.87$ & $<0.001^{*}$ \\
\hline Perifovea & $53.09 \pm 6.76$ & $49.13 \pm 6.14$ & $<0.001^{*}$ & $52.88 \pm 6.72$ & $47.69 \pm 5.92$ & $<0.001^{*}$ \\
\hline Perifovea-superior-hemi & $53.55 \pm 6.70$ & $49.64 \pm 6.25$ & $<0.001^{*}$ & $53.44 \pm 6.63$ & $48.39 \pm 6.21$ & $<0.001^{*}$ \\
\hline Perifovea-Inferior-hemi & $52.66 \pm 7.06$ & $48.61 \pm 6.34$ & $<0.001^{*}$ & $52.31 \pm 7.10$ & $47.03 \pm 5.99$ & $<0.001^{*}$ \\
\hline Perifovea-tempo & $55.29 \pm 6.10$ & $51.87 \pm 5.42$ & $<0.001^{*}$ & $55.32 \pm 5.99$ & $50.46 \pm 5.24$ & $<0.001^{*}$ \\
\hline Perifovea-superior & $52.93 \pm 7.39$ & $48.75 \pm 6.92$ & $<0.001^{*}$ & $52.68 \pm 7.17$ & $47.50 \pm 7.17$ & $<0.001^{*}$ \\
\hline Perifovea-nasal & $52.46 \pm 7.36$ & $48.35 \pm 6.75$ & $<0.001^{*}$ & $52.15 \pm 7.35$ & $46.95 \pm 6.43$ & $<0.001^{*}$ \\
\hline Perifovea-inferior & $51.73 \pm 7.67$ & $47.53 \pm 7.10$ & $<0.001^{*}$ & $51.48 \pm 7.76$ & $45.97 \pm 6.54$ & $<0.001^{*}$ \\
\hline \multicolumn{7}{|c|}{ VD of FD-300 $(\%)$, mean $\pm S D$} \\
\hline FD-300 Area Density & $53.33 \pm 5.11$ & $53.02 \pm 4.54$ & 0.476 & $53.27 \pm 5.11$ & $52.00 \pm 5.24$ & $0.038^{*}$ \\
\hline FD-300 Length Density & $12.30 \pm 1.91$ & $11.98 \pm 1.56$ & $0.039^{*}$ & $12.23 \pm 1.98$ & $11.49 \pm 1.98$ & $0.002 *$ \\
\hline \multicolumn{7}{|c|}{$\begin{array}{l}\text { RNFL thickness in the RPC }(\mu \mathrm{m}) \text {, } \\
\text { mean } \pm \text { SD }\end{array}$} \\
\hline Peripapillary & $113.70 \pm 14.07$ & $114.60 \pm 13.86$ & 0.471 & $115.86 \pm 12.74$ & $113.70 \pm 15.12$ & 0.192 \\
\hline Superior-hemi & $114.09 \pm 14.80$ & $115.21 \pm 16.47$ & 0.426 & $116.70 \pm 13.76$ & $114.66 \pm 15.50$ & 0.239 \\
\hline Inferior-hemi & $113.28 \pm 15.34$ & $113.95 \pm 14.21$ & 0.609 & $114.95 \pm 13.50$ & $112.66 \pm 16.78$ & 0.202 \\
\hline Tempo & $79.35 \pm 11.71$ & $76.92 \pm 10.38$ & $0.014^{*}$ & $78.70 \pm 10.77$ & $77.37 \pm 10.31$ & 0.286 \\
\hline Superior & $137.0 \pm 20.38$ & $138.54 \pm 22.08$ & 0.442 & $140.49 \pm 19.43$ & $137.61 \pm 20.24$ & 0.220 \\
\hline
\end{tabular}


(Cont. Supplemental Table 2)

\begin{tabular}{|c|c|c|c|c|c|c|}
\hline & \multicolumn{3}{|c|}{ Training set $(n=508)$} & \multicolumn{3}{|c|}{ Held-out validation set $(n=287)$} \\
\hline Nasal & $98.20 \pm 16.89$ & $100.13 \pm 17.36$ & 0.208 & $100.86 \pm 16.64$ & $99.64 \pm 18.41$ & 0.557 \\
\hline Inferior & $145.12 \pm 22.24$ & $147.47 \pm 20.88$ & 0.219 & $148.03 \pm 20.15$ & $144.96 \pm 24.51$ & 0.246 \\
\hline Tempo-superior & $82.74 \pm 12.61$ & $79.53 \pm 11.60$ & $0.003^{*}$ & $82.40 \pm 11.87$ & $80.07 \pm 12.51$ & 0.107 \\
\hline Superior-nasal & $137.43 \pm 26.43$ & $142.79 \pm 28.69$ & $0.029^{*}$ & $140.90 \pm 25.12$ & $141.79 \pm 26.78$ & 0.770 \\
\hline Nasal-superior & $106.34 \pm 19.14$ & $109.89 \pm 21.03$ & $0.049^{*}$ & $109.71 \pm 19.45$ & $109.27 \pm 21.10$ & 0.854 \\
\hline Nasal-inferior & $88.01 \pm 17.30$ & $87.91 \pm 16.72$ & 0.948 & $89.79 \pm 16.77$ & $87.60 \pm 18.89$ & 0.300 \\
\hline Inferior-nasal & $141.27 \pm 28.20$ & $143.95 \pm 26.93$ & 0.276 & $144.82 \pm 26.29$ & $141.94 \pm 30.28$ & 0.390 \\
\hline Inferior-tempo & $150.02 \pm 26.31$ & $151.95 \pm 23.77$ & 0.386 & $152.09 \pm 23.32$ & $148.78 \pm 26.10$ & 0.258 \\
\hline Whole Image Capillary & $48.72 \pm 3.26$ & $48.86 \pm 2.77$ & 0.615 & $49.42 \pm 2.91$ & $48.15 \pm 3.38$ & $0.001^{*}$ \\
\hline Inside Disc All & $60.46 \pm 4.56$ & $60.36 \pm 3.84$ & 0.785 & $60.30 \pm 4.36$ & $58.76 \pm 4.63$ & $0.004^{*}$ \\
\hline Inside Disc Capillary & $51.04 \pm 5.44$ & $50.64 \pm 4.70$ & 0.374 & $50.74 \pm 5.43$ & $49.29 \pm 5.24$ & $0.022^{*}$ \\
\hline Peripapillary All & $57.47 \pm 3.85$ & $57.87 \pm 3.39$ & 0.217 & $58.41 \pm 3.32$ & $57.22 \pm 4.18$ & $0.008^{*}$ \\
\hline Peripapillary Capillary & $50.86 \pm 3.73$ & $51.26 \pm 3.29$ & 0.194 & $52.03 \pm 3.32$ & $50.72 \pm 4.19$ & $0.003^{*}$ \\
\hline Superior-hemi All & $57.87 \pm 4.10$ & $58.42 \pm 3.53$ & 0.103 & $58.93 \pm 3.41$ & $57.85 \pm 4.26$ & $0.018^{*}$ \\
\hline Inferior-hemi All & $57.05 \pm 3.85$ & $57.27 \pm 3.69$ & 0.499 & $57.84 \pm 3.48$ & $56.53 \pm 4.40$ & $0.005^{*}$ \\
\hline Superior-hemi Capillary & $50.94 \pm 4.06$ & $51.55 \pm 3.54$ & 0.074 & $52.33 \pm 3.54$ & $51.07 \pm 4.41$ & $0.008^{*}$ \\
\hline Inferior-hemi Capillary & $50.76 \pm 3.79$ & $50.95 \pm 3.65$ & 0.562 & $51.70 \pm 3.44$ & $50.31 \pm 4.50$ & $0.003^{*}$ \\
\hline Nasal-superior & $47.20 \pm 4.80$ & $47.94 \pm 4.42$ & 0.069 & $48.53 \pm 4.34$ & $47.73 \pm 5.44$ & 0.168 \\
\hline
\end{tabular}

Abbreviations: CAD, coronary artery disease; VD, vessel density; SCP, superficial capillary plexus; DCP, deep capillary plexus; FD, foveal density; RNFL, retinal nerve fiber layer; RPC, radial peripapillary capillary.

${ }^{*} p<0.05$ is considered statistically significant. 


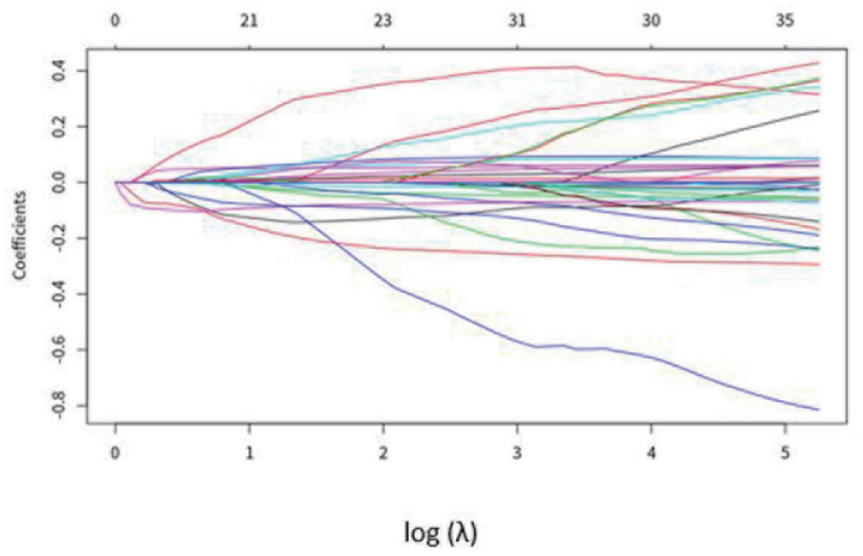

(A)

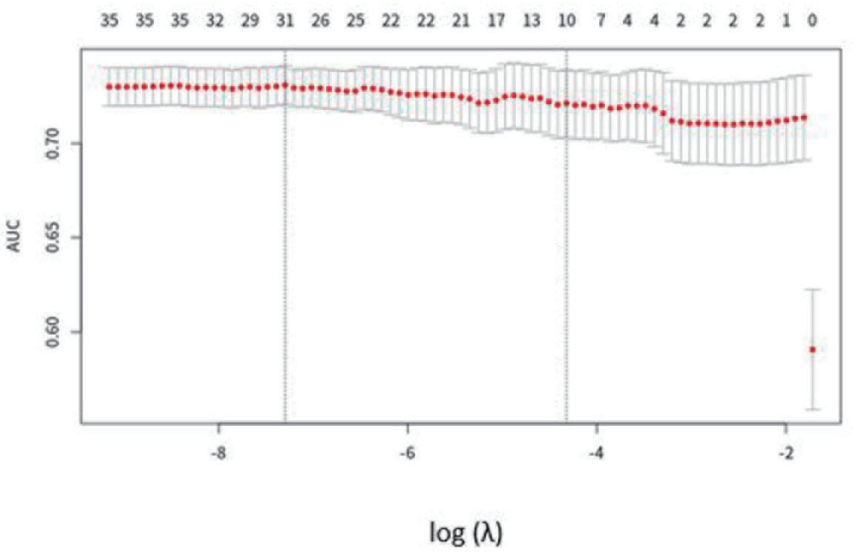

(B)

Supplemental Fig. 3. Features selection using the LASSO binary logistic regression model

(A) Tuning parameter $(\lambda)$ selection in the LASSO model used 10-fold cross-validation via minimum criteria. The area under the curve (AUC) was plotted versus $\log (\lambda)$. A $\lambda$ value of 0.0133 , with the $\log (\lambda)$ of -4.33 was chosen according to 10 -fold cross-validation. (B) A coefficient profile plot was produced against the $\log (\lambda)$ sequence. A vertical line was drawn at the value selected using 10 -fold cross-validation.

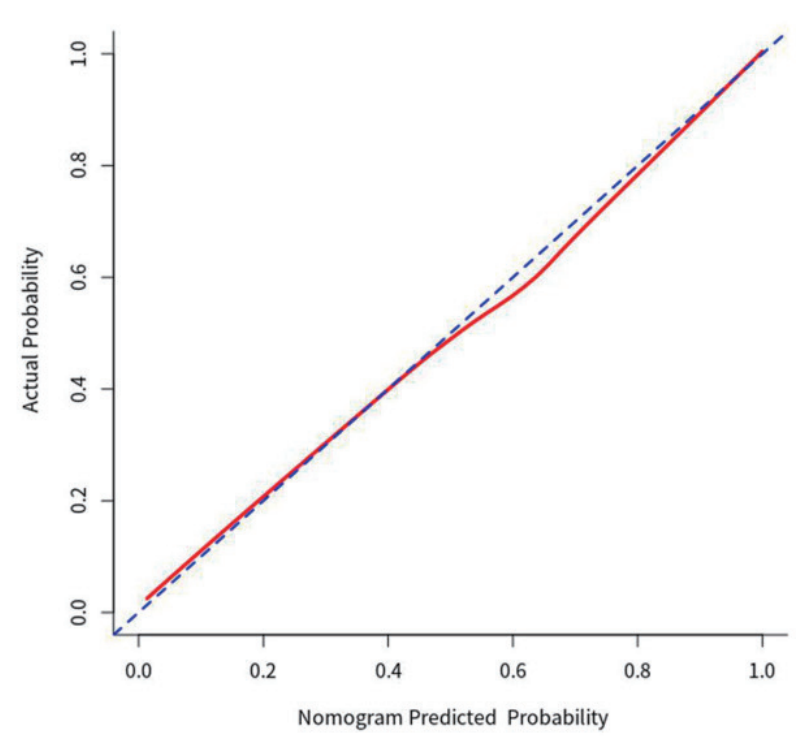

(A) Training set

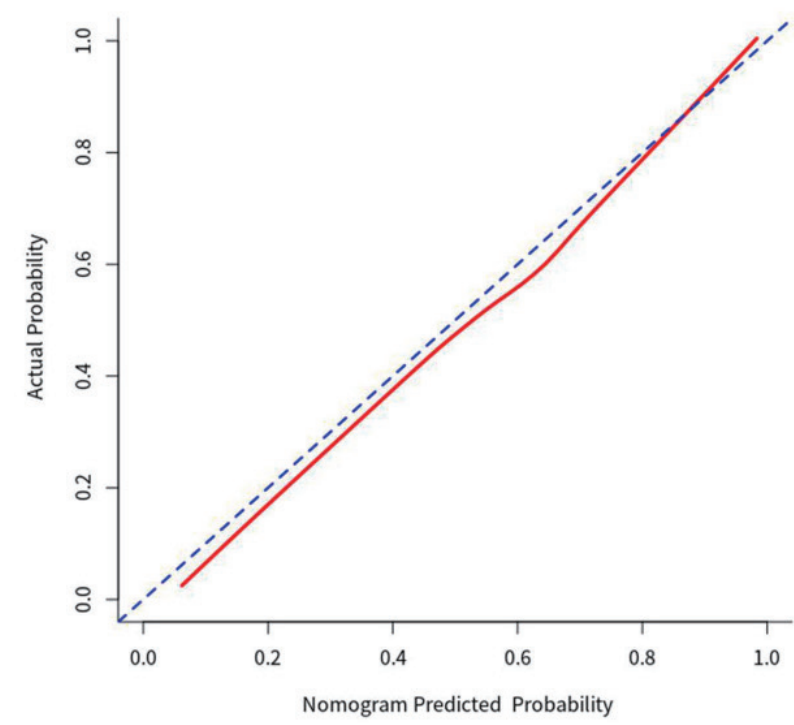

(B) Held-out validation set

Supplemental Fig.4. Calibration curves of the nomogram in two data sets

Calibration curves of the retinal vasculature nomogram in the (A) training and (B) held-out validation sets.

For the calibration curve, the $\mathrm{Y}$-axis represents the actual presence of CAD, and the X-axis represents the predicted presence of CAD risk. The solid red line represents the performance of the nomogram, where a closer fit to the diagonal dotted line represents a better prediction.

Abbreviation: CAD, coronary artery disease. 